\title{
Advances in RNA nanotechnology promise to transform medicine
}

\section{The Second International Conference on RNA Nanotechnology and Therapeutics Lexington, KY, USA, 3-5 April 2013}

The second International Conference on RNA Nanotechnology and Therapeutics was held on the 3-5 April in Lexington, (KY, USA). The focus of the conference was on leveraging the unique chemical and biological properties of RNA to promote transformative advances in medicine. The conference convened more than 200 researchers from 15 countries and many disciplines, roughly double the participants of the first conference. While many presentations focused on the design, assembly and characterization of RNA nanoparticles and their uses for in vivo and in vitro sensing, diagnosis and therapy, others covered a variety of relevant areas of RNA biology and chemistry.

The Second International Conference on RNA Nanotechnology and Therapeutics was organized to build on the success of the First International Conference, held in 2010 in Lexington, OH, USA [1,101]. Conference chair Peixuan Guo (University of Kentucky, KY, USA) provided an overview of the unique advantages of RNA nanoparticles (NPs), their applications in medicine and approaches for overcoming challenges that limit their use such as RNase sensitivity, endosome escape, low cell permeability and thermodynamic instability [102]. Oral sessions were organized around: design, construction and assembly of RNA NPs; RNA dynamics and intermolecular interactions; delivery of RNA NPs to cells; and intercellular transport of RNA in extracellular vesicles (EVs; exosomes). Notably, the conference brought together, for the first time, scientists studying RNA transfer in EVs and developers of synthetic RNA NPs.

\section{Keynotes}

RNA nanotechnology exploits the modularity and designability of RNA at multiple structural levels (secondary, tertiary and quaternary) to rationally design and functionalize NPs for diverse applications [2]. Keynote speakers David Lilley (University of Dundee, Dundee, UK) and Eric Westhof (Université de Strasbourg, Strasbourg, France) elucidated structural, energetic and dynamic aspects of RNA modularity at the level of base-pairing interactions and specific 3D motifs, such as the kink-turn, a motif that introduces sharp bends in RNA double helices. Rob Batey (University of Colorado, CO, USA) reported on the modularity of natural riboswitches, demonstrating that their aptamer domains and expression platforms can be separated and recombined by mixing and matching to design new RNA-based genetic switches, and their architectures repurposed through in vitro selection to evolve new specificities. Conference co-chair, Neocles Leontis (Bowling Green State University, OH, USA), described the comprehensive, searchable online resource RNA Motif Atlas, which extracts RNA 3D motifs from atomicresolution structures and organizes them by geometric similarity [103].

\section{Technological advances}

Significant technical advances in the synthesis, manipulation and characterization of RNA in the production of useful NPs were presented. Subha Das (Carnegie Mellon University, PA, USA) described the use of 'click chemistry' to specifically modify, label and derivatize RNA for a range of applications, including delivery of siRNA to cells. David Gorenstein (University of Texas, TX, USA) presented the use of 'splitpool,' bead-based combinatorial libraries and bead selection technology to obtain 'X-aptamers', incorporating mono- and di-thiophosphates in the backbone for nuclease resistance, and a range of organic and amino acid-like modifications at the 5-position of 2'-deoxyuridine to improve affinity and specificity of in vivo targeting. Gang Han (University of Massachusetts Medical School, MA, USA) presented biocompatible upconversion luminescent NPs, which make use of lanthanide luminescence for in vivo imaging. These NPs are extremely photostable, free of

\section{Neocles Leontis*1, Blake Sweeney ${ }^{1}$, Farzin Haque ${ }^{2}$ \& Peixuan Guo ${ }^{2}$}

${ }^{1}$ Department of Chemistry, Bowling Green State University, Bowling Green, $\mathrm{OH} 43403$, USA

${ }^{2}$ Nanobiotechnology Center, Markey Cancer Center \& Department of Pharmaceutical Sciences, College of Pharmacy, University of Kentucky, Lexington, KY 40536, USA

*Author for correspondence:

leontis@bgsu.edu 
autofluorescence and 'blinking', and exhibit outstanding tissue penetration with minimal light scattering. Zhen Huang (Georgia State University, GA, USA) demonstrated the advantages of RNA-containing selected selenium-modified bases to facilitate $\mathrm{x}$-ray crystallography of RNA.

Early detection and diagnosis are crucial for treating many types of cancer, including lung and colorectal cancer, and small regulatory RNAs called miRNAs are emerging as useful cancer biomarkers. Linquin Gu (University of Missouri, MO, USA) reported on the use of nanopore arrays for rapid, high-throughput, sensitive, label-free miRNA detection requiring no amplification. Ming Hammond (University of California, Berkeley, CA, USA) described a microfluidic screening platform for studying small moleculedependent RNA conformational changes. Francesca Storici (Georgia Institute of Technology, GA, USA) reported that RNA transcripts can serve as templates to repair damaged DNA and can directly transfer genetic information to chromosomal DNA without being first converted to cDNA. These findings from basic biology provide new avenues for designing therapeutic RNAs that can potentially get to the root of genetic diseases by allowing for gene therapy and genome engineering.

\section{Computational support for RNA nanotechnology}

To advance RNA nanotechnology, we need to meet two complementary computational challenges: predicting the 3D structures of cellular RNAs from sequence (the RNA folding problem) and designing RNA sequences to fold into specific structures (the 'reverse folding' problem). These challenges are difficult because even structured RNAs contain flexible regions that change conformation in response to changes in the environment and binding of ligands. A number of speakers, including co-chair Bruce Shapiro (National Cancer Institute, MD, USA), Kirill Afonin (National Cancer Institute), Nikolay Dokholyan (University of North Carolina, NC, USA) and Rhiju Das (Stanford University, CA, USA) addressed aspects of these challenges. Shapiro and Afonin successfully predicted the reassembly kinetics and thermodynamics of hybrid RNA/DNA NPs with split functionalities that show gene silencing in vivo. Rhiju Das presented EteRNA [104], an online game that recruits 'citizen scientists' to discover new RNA folding rules through multiple rounds of play, aided by feedback from high-throughput chemical probing of user-submitted RNA designs. Dokholyan presented coarse-grained methods for rapid conformation searching to predict 3D structures of large RNAs.

\section{Extracellular vesicles}

Conference participants received a crash course in the exciting area of EVs (also called exosomes, 'microvesicles' or 'shedding vesicles'). The term 'extracellular vesicles' is preferred to avoid confusion with cellular components involved in RNA degradation that are also called exosomes [3]. EVs are nanovesicles of endocytic origin and are 40-100 $\mathrm{nm}$ in diameter. They are released by many different types of cells for intercellular communication, and regulation of growth and differentiation; they contain specific subsets of host cell proteins, RNA and lipids.

Douglas Taylor (University of Louisville, KY, USA) described isolation of RNA cargo from exosomes to provide 'liquid biopsies' for cancer diagnosis, an approach that does not require knowing the cancer's actual location in the body. Stefano Pluchino (Cambridge University, Cambridge, UK) introduced the concept of stem cell therapeutic plasticity to refer to how neural stem/precursor cells protect the CNS from chronic inflammationdriven degeneration. They do so, not only by replacing lost or damaged cells, but eventually by engaging in sophisticated programs of intercellular communication involving the transfer of EVs from donor neural stem/precursor cells to target cells. Preliminary data suggest that EV-mediated transfer of mRNAs and proteins affect gene and protein expression in target cells, and constitute a major mechanism of functional instruction of target cells by neural stem/precursor cells. This new understanding of stem cell function is relevant to immunology and cancer biology, and holds the potential for revolutionizing how we treat chronic and degenerative diseases.

Melissa Piper (Ohio State University, $\mathrm{OH}$, USA) described how EVs function to maintain homeostasis in healthy 
individuals through the action of the packaged proteins, and coding and noncoding RNAs. Her in vitro results demonstrated that EVs transfer RNA molecules to a variety of different recipient cell types and that myeloid-derived EVs mediate genetic changes, leading to the production of mature and functional phagocytic macrophages. Work on EVs has the potential to reshape both how we define sickness and identify health.

\section{Delivery of siRNAs \& other therapeutic RNAs}

A number of presentations addressed in vivo delivery of RNA NPs carrying siRNA payloads. Kato Shum (City of Hope National Medical Center, CA, USA) and John Rossi (City of Hope National Medical Center) reported dual function therapeutic aptamer-siRNA NPs based on multivalent 'RNA aptamer-stick-siRNA' constructs to specifically deliver siRNAs to target cells at therapeutic doses, while avoiding unwanted off-target side effects. Targets include lymphoma cells and HIV-1-infected cells. This work demonstrated that cell-targeting aptamers can serve as delivery vehicles for siRNAs or other RNA therapeutics.

Xiaoting Zhang (University of Cincinnati, OH, USA) discussed new approaches for dealing with the problem of resistance to tamoxifen therapy in breast cancer patients. The monoclonal antibody drug Herceptin ${ }^{\circledR}$ (trastuzumab; Genentech, CA, USA) blocks HER2, a receptor tyrosine kinase. Transcriptional coactivator MED1, a component of the mediator complex, plays a key role in HER2-mediated tamoxifen resistance. Zhang described the development of RNA aptamers to disrupt the interactions between estrogen receptor and MED1, as well as pRNA nanotechnology-based siRNA delivery to diminish MED1 protein expression so as to overcome endocrine resistance in human breast cancer.

Jorgen Kjems (Aarhus University, Aarhus, Denmark) reported a number of new siRNA delivery systems, including encapsulation in chitosan nanoplexes. Successful targeting and knockdown of therapeutically important genes was achieved in mice using intravenous injection. In addition, Kjems reported new types of circular RNA molecules that act as highly efficient miRNA sponges in neurons. Plasmid-based vectors capable of expressing circular RNA sponges in mammalian cells were designed and demonstrated to effectively regulate miRNA-7 activity. These vectors may represent new drug platforms that can be redesigned to treat other miRNA-associated diseases.

\section{Gene therapy}

Several talks focused on gene therapy. Noting that a major bottleneck in human gene therapy is a deficit of safe and efficient methods for delivering DNA to target cells, Daniel Pack (University of Kentucky) described 'nanobiovectors' assembled from viral and synthetic components. By leveraging the advantages of recombinant viruses for efficient gene delivery and genomic integration, and those of synthetic polymers for efficient escape from endocytic vesicles, these materials have the potential to revolutionize the treatment of diseases, ranging from cystic fibrosis to cardiovascular disease.

\section{Self-assembling RNA NPs for cancer therapy}

Guo's team, including Farzin Haque and Hui Zhang (University of Kentucky, KY, USA), described biophysical, crystallographic, single-molecule and 3D modeling studies of the structure, stoichiometry and assembly of the hexameric pRNA, a component of the Phi29 viral DNA packaging motor. The Guo group exemplified the development of a RNA 'toolkit' consisting of a modular hairpin, internal and junction loops derived from structured RNAs (i.e., the pRNA) for use as building blocks and rules for combining them. This approach makes it possible to design RNA monomers capable of self-assembly to form a diverse range of NP architectures, ranging from dimers to heptamers. Among branching architectures, the pRNA-3WJ and pRNA-X motifs are thermodynamically and chemically stable, efficiently selfassemble in the absence of divalent salts, are resistant to RNase or denaturation by eight molar urea, and, therefore, are stable in serum for $>36 \mathrm{~h}$ after systemic injection in vivo. The NP architectures allow incorporation of diverse functionalities (siRNA, miRNA, ribozymes and aptamers), do not 
induce cytokines, IFN-I, antibodies or toxicity in mice, and allow in vivo targeting of cancer cells without accumulation in normal organs and tissues. Pharmacokinetic analysis revealed half-lives extended 100-fold compared with simple siRNA. These findings open new areas for cancer targeting and treatment. The versatility and diversity in structure and function derived from a limited toolkit from one biological RNA molecule suggests the immense potential of RNA NPs in diagnostic and therapeutic applications.

\section{Conclusion}

Space allows only a summary of selected highlights of the conference, the choice of which is necessarily subjective, so readers are urged to consult the references to the conference program for information on many other interesting presentations on related topics [105]. In conclusion, the conference highlighted how the rapid advance of RNA chemistry, biophysics and basic biology is creating many new opportunities for translating basic science into clinical practice. Furthermore, we are gaining a better understanding of the role of RNA in maintaining health so as to advance the prevention as well as the treatment of disease.

\section{Financial \& competing interests disclosure}

N Leontis holds patents in RNA self assembly. P Guo is a cofounder of Kylin Therapeutics, Inc., and
Biomotor and Nucleic Acid Nanotechnology Development Corp. Ltd. The authors have no other relevant affliations or financial involvement with any organization or entity with a financial interest in or financial conflict with the subject matter or materials discussed in the manuscript apart from those disclosed.

No writing assistance was utilized in the production of this manuscript.

\section{References}

1 Shukla GC, Haque F, Tor Y. A boost for the emerging field of RNA nanotechnology. ACS Nano 5, 3405-3418 (2011).

2 Guo P. The emerging field of RNA nanotechnology. Nat. Nanotechnol. 5 , 833-842 (2010).

3 Gould SJ, Raposo G. As we wait: coping with an imperfect nomenclature for extracellular vesicles. JEV2, 20389 (2013).

\section{Websites}

1012010 International Conference of RNA Nanotechnology and Therapeutics. www.eng.uc.edu/nanomedicine/RNA2010

1022013 International Conference of RNA Nanotechnology and Therapeutics. http://nanobio.uky.edu/RNA2013/

103 RNA 3D Hub. RNA 3D motif atlas. http://rna.bgsu.edu/rna3dhub/motifs

104 EteRNA. http://eterna.cmu.edu/web/

105 Second International Conference of RNA Nanotechnology and Therapeutics (2013). http://nanobio.uky.edu/RNA2013/Program_ booklet-2nd_Intl_Conf_on_RNA_Nano_ and_Ther.pdf 